\title{
Vinylsiloxanether: A New Impression Material. Clinical Study of Implant Impressions with Vinylsiloxanether versus Polyether Materials
}

\author{
Norbert Enkling, $\cdots ; ;^{\star}$ Stefan Bayer, $\cdots ;^{\dagger}$ Peter Jöhren, $\cdots ; ;^{\ddagger}$ Regina Mericske-Stern, $\cdots \lessgtr$
}

\begin{abstract}
Aim: The performance of three elastomeric materials for the open monophase implant impressions technique was tested under the following clinical conditions: polyether (IM), and vinylsiloxanether without (ID) and with additional simultaneous splinting of the implant impression copings with a higher shore hardness A-silicone (IDF).

Materials and Methods: The three test groups, IM, ID, and IDF, were randomly allocated 10 test subjects with three to five implants each. The impressions were analyzed regarding the subjective clinical assessments with 11-point rating scales by the dentist, the patient, and the dental technician, as well as to the comparison of these assessments with the objectified clinical fit of the manufactured crowns based on standard clinical evaluation criteria. The three groups were statistically analyzed on the basis of the hypothesis of non-inferiority of ID, IDF versus IM (alpha $<0.05$ ).

Results: The results of the study showed the objective clinical fit of the dental prostheses made using ID being comparable to the results obtained with IM. Compared with ID and IM, the precision of fit attained with IDF was reduced, although the subjective dentist assessments of IDF were in parts significantly better than those of IM and ID $(p=.015)$. A statistically significant superiority of ID in comparison with IM could be determined with regard to the subjective ratings of the taste by the test subject $(p<.01)$, of the handling $(p<.001)$ and of the precision details of impression $(p=.012)$ by the dentist, and of removing the plaster model from the mold by the dental technician $(p=.017)$.

Conclusions: The overall results of the vinylsiloxanether material in terms of the patients', dentists', and dental technicians' assessments proved to be equivalent or superior to those of the polyether material. The IDF technique cannot be recommended for this application.
\end{abstract}

KEY WORDS: implant impressions, polyether, randomized clinical study, splinting of implant copings, vinylsiloxanether

\section{INTRODUCTION}

The materials available on the market to date are not perfectly appropriate for the creation of identical replicas of the actual oral scenario in model form. ${ }^{1,2}$ Dental

*.•, Department of Prosthodontics, University of Bern, Bern, Switzerland; ${ }^{+\bullet}$, Department of Prosthodontics, Preclinical Education and Dental Materials Science, University of Bonn, Bonn, Germany; ${ }^{* \bullet}$, Department of Oral Surgery, University of Witten/Herdecke, Witten,

2 Germany; ${ }^{\varsigma} \cdot$, Department of Prosthodontics, University of Bern, Bern, Switzerland

Reprint requests: Dr. Norbert Enkling, Department of Prosthodontics, University of Bern, Freiburgstrasse 7, CH-3010 Bern, Switzerland; e-mail: norbert.enkling@zmk.unibe.ch

(c) 2009, Copyright the Authors

Journal Compilation @ 2009, Wiley Periodicals, Inc.

DOI 10.1111/j.1708-8208.2009.00240.x prostheses that fit the master model perfectly do not always allow for tension-free integration into the patient's mouth. Intraoral impressions, which are used in the production of dental prostheses on implants, have to meet highly exacting standards in terms of their precision. Potential transfer errors as a result of discrepancies between the oral and model situation may prevent the materialization of a passive fit between the implants and supra-construction that leads to the presence of permanent forces that adversely affect the interface between implant and bone. The long-term effects of such situations cannot be assessed at this time. ${ }^{3-5}$

The suggested materials for implant impressions include A-silicones, C-silicones, polyether materials, polysulfide materials, hydrocolloids, and impression plaster. ${ }^{6-13}$ Depending on the implant system used, the implant impressions can be created using the open or 
closed impressions technique, and a custom or stock impression trays. ${ }^{11,12,14-20}$

When taking impressions of several neighboring implants, a primary splinting of the implant impression copings is being considered with the objective of increasing the impression's precision. This can be achieved by creating a splinting with self-cured acrylic resins, dually hardening plastics, plaster, or by creating a direct connection between the impression copings and the impression tray with a self-cured acrylic resin. ${ }^{19,21-25}$

However, the benefits of the reinforced splinting may be negatively affected by the shrinkage of the selfcured acrylic resin and the resulting deformation of the impression. ${ }^{26-28} \mathrm{~A}$ splinting of the impression copings with a self-cured acrylic resin may also cause the temperature in the implant shoulder to rise by an average of 4 to $5^{\circ} \mathrm{C}$, which can consequently lead to biologic damages to the implant-bone interface. ${ }^{29}$ Consequently, most users forego the utilization of an additional splinting as a rule. Instead, they ensure that the impression coping is framed like a chimney at a clearance of 2 to $5 \mathrm{~mm}$ from the custom tray so that the fixation of the coping in the optimum layer thickness of the impression material is warranted. . $^{8,12,17,18,30,31}$

As an alternate, it has been proposed to achieve an improved fixation of the impression coping in the impression by way of a splinting using A-silicone of a higher shore grade, for example, in the form of a slowly setting registration material, simultaneously with the taking of an impression with a low shore grade medium fluxionary impression material..$^{32}$ Given that this process results in the creation of a chemical bond between the registration materials for the splinting and the impression materials, and considering the fact that the dimensional variations of these registration materials are similarly minimal as those of the impression materials, ${ }^{28}$ this approach appears to be expedient.

Among the numerous differing impression procedures, the current established gold standard is the open impressions technique. It uses a stable custom tray and the polyether material "Impregum penta" (3M Espe Company, Seefeld, Germany), and is the standard procedure used by many dentists performing implants. ${ }^{3,6,12}$

In 2009, the Kettenbach Company (Eschenburg, Germany) launched a new impression material called vinylsiloxanether "Identium" into dental markets. It is a chemical combination of a polyether material and a 8 polyvinylsiloxane, that is, A-silicone. According to infor- mation provided by the Kettenbach Company, the combination of the polyether material with polyvinylsiloxane components introduces theoretical advantages, given that it does maintain similar mechanical and hydrophilic properties while achieving its final hardness more expeditiously. Moreover, it is possible to create a chemical bond between vinylsiloxanether and polyvinylsiloxane.

Along with material science parameters, the decisions made in routine practical applications when it comes to the pros and cons of using a certain material are contingent upon the subjective assessments of the material by not only the dentist, but also by the patient and the dental technician. ${ }^{33}$

\section{MATERIALS AND METHODS}

\section{Groups of Test Subjects}

A total of 30 test subjects (15 men, 15 women) with neighboring 3-5 SIC-Ace implants (SIC Invent AG, Basel, Switzerland) in the lower side tooth region with remaining natural teeth were recruited for the clinical study. In all cases, the open impression technique with custom trays was used, and the implants were to be supplied with cemented single-tooth crowns based on standard titanium abutments (SIC No. 936163).

The allocation of the 30 test subjects to the three impression groups was randomized so that each group consisted of five women and five men:

1. ID: using vinylsiloxanether "Identium Medium" (Kettenbach Company)

2. IDF: using vinylsiloxanether "Identium Medium" (Kettenbach Company) with additional simultaneous splinting of the impression copings with the A-silicone "Futar D Slow" (Kettenbach Company)

3. IM: using polyether "Impregum penta" (3M Espe Company)

\section{Clinical Protocol}

The clinical situation was standardized insofar that the implants of all 30 test subjects had been surgically and uniformly exposed after a healing period of 3 months and 14 days prior to the impression taking. All test subjects had impressions taken by one dentist within a week thereafter. Seven days after the impression had been taken, the crown abutments were tested with occluding self-cured acrylic resin stops for intraoral checkups of the intra-articulated situations. If necessary, the 
occluding stops were adjusted and the upper jaw model was newly intra-articulated. After another 7 days, the fully ceramic veneered single crowns had been manufactured. The laboratory work was performed in a standardized manner by two dental technicians. Another dentist, who was given no information as to the impression material used, performed the clinical fit testing of the implant dental prostheses within a week. The dentists and dental technicians participating in the study had been calibrated as to the completion of the questionnaires before the study began. The dentists and dental technicians provided their evaluations independently and separately.

The mixed impressions (implants and natural teeth) were implemented using an open impressions technique with custom trays and screwed in impression copings. The impression materials "Identium" and "Impregum" were both prepared straight from the polyester bag using the automated mixing device - Pentamix 2 (3M Espe Company). Material "Futar D Slow" was prepared in a dual-chamber syringe using a mixing gun (Kettenbach Company). Before the tray was guided into the test subject's mouth, the implant area and the natural teeth were additionally injection surrounded with "Identium" or

12 "Impregum." Impressions of the opposing dentitions were taken with a metal stock tray and alginate. All models were made of type IV super hard plaster (Esthetik Base $300^{\circledR}$, dentona AG, Dortmund, Germany).

The groups were compared in terms of the subjective clinical assessments by the dentist and the patient concerning the impression; the assessment by the dental technician was in conjunction with the model creation and in reference to clinical fit of the implanted dental prosthesis, which had been manufactured on the basis of 13 the impression.

\section{Subjective Assessments}

The assessment of the impressions by the respective dentists, patients, and dental technicians was based on numerical 11-point rating scales $(0-10)$. The respective 11-point rating scales ranged from negative (0) to 14 positive (10).

The test subjects were asked to rate the taste of the implant impression material and the alginate. The dentists were asked to perform subjective evaluations of the handling, detail precision of the impression material, and the quality of the impression. They also recorded the time it took to make the impression. The dental technician was asked to subjectively evaluate the rotation safety of the impression coping in the impression while screwing in the laboratory analogue. Dental technicians also rated the hydrophilic characteristics of the material during plaster casting, the ease of removing the plaster model from the mold, and the detail precision of the plaster model.

\section{Objective Clinical Evaluation}

In the final objective checkup, the model situation was compared with the clinical situation. For this procedure, the screwed-on abutments were used to manufacture a control key made of "Pattern Resin" (GC, Tokyo, Japan) on the master model. This key was tested for a vertical and horizontal tension-free fit in the patient's mouth. The optional responses were "Yes" and "No." The approximate fit of the implant crowns was verified using a $0.05 \mathrm{~mm}$ metal template and rated as either "cannot be inserted = approximate contact force too strong," "can be inserted under pressure=adequate," and "can be inserted without pressure $=$ too weak."

The interocclusal fit of the implant crowns was rated in terms of the required adjustments and allocated to categories "good" (no adjustments required), "moderate" (drilling required to improve fit $<50 \mu \mathrm{m}$ ), and "bad" (non-occlusion, drilling requirements exceed $\geq 50 \mu \mathrm{m})$.

Ethical approval for the study was obtained from the Ethics Committee of the Faculty of Dental Medicine, University of Witten/Herdecke, Germany.

The results were statistically analyzed for noninferiority of ID, IDF versus IM using the Wilcoxon rank sum test, and for testing the occlusal, horizontal, and vertical fit of the crowns using the Fisher's exact test $($ alpha $<0.05)$.

\section{RESULTS}

\section{Subjective Assessments}

Test Subject/Patient. Material alginate, which was used as a taste comparison reference by the groups, was rated as largely positive by all three groups. The test subjects also perceived the taste of ID and IDF as rather positive, while IM received substantially more negative ratings $(p<.002)$ (see Table 1$)$.

Dentist. In terms of handling, the dentists rated ID and IDF as statistically significantly better than IM 


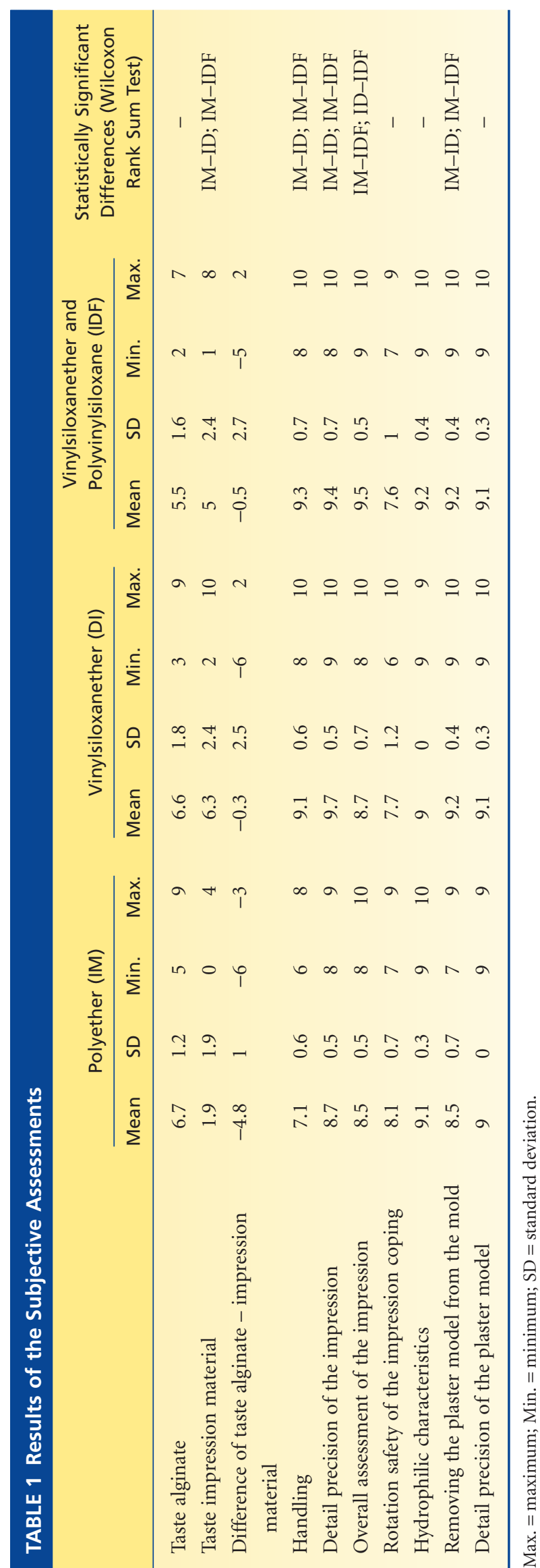

( $p=.0001)$. The results obtained by ID and IDF were similar in terms of handling. In reference to the detail precision of the impression, all three groups received "excellent" ratings, whereby in comparison with IM, ID $(p=.012)$ and IDF $(p=.0235)$ were found to be superior. ID and IDF were rated equal. In the overall assessment of the impression, all three methods were rated as excellent, whereby IDF was found to be superior to IM $(p=.0024)$ and to ID $(p=.0151)$. IM and ID were found to be similar. The time that had to be expended on the performance of the impressions was comparable and totaled an average of 8 to 10 minutes.

Dental Technicians. No differences between the plaster models were found as far as the detail precision of the impression materials subjectively assessed by the dental technicians is concerned. All methods yielded model surfaces with high detail precision. Moreover, the dental technicians found the impression copings' rotation resistance in the impression material while screwing in the laboratory analogues equally good in all cases. In terms of the impression materials' hydrophilic properties, which were evaluated on the basis of the plaster casting performance of the impressions, all materials were also found to perform similarly well. The posthardening removal of the plaster model from the impression appeared to be significantly easier on the impressions using ID and IDF $(p=.0172)$ than those rated for IM; however, all groups received a rating of "good" to "excellent." No differences were found between ID and IDF during this evaluation.

\section{Objective Evaluations of the Clinical Precision}

The clinical fit of the control key, which had been manufactured on the abutments of the master model, did not reveal any differences between the groups in the vertical relation of the clinical application. In the horizontal relation, the ID demonstrated a superior fit than IM, and the latter was superior to IDF; however, only the differences between ID and IDF reached a statistically significant level ( $p=.0253)$ (see Tables 2 and 3).

In terms of the approximate contact strength of the crowns produced, no differences could be found in categories "too strong" or "adequate" approximate contacts. However, in the category of approximate contacts that are too weak, a statistically significant number was found with IM in comparison with ID $(p=.0057)$ and in comparison with IDF $(p=.0399)$. 
TABLE 2 Results of the Objective Evaluations 1

\begin{tabular}{|c|c|c|c|c|}
\hline & $\begin{array}{l}\text { Polyether } \\
\text { (IM) }\end{array}$ & $\begin{array}{l}\text { Vinylsiloxanether } \\
\text { (ID) }\end{array}$ & $\begin{array}{l}\text { Vinylsiloxanether and } \\
\text { Polyvinylsiloxane (IDF) }\end{array}$ & $\begin{array}{c}\text { Statistically Significant } \\
\text { Differences (Fisher's } \\
\text { Exact Test) }\end{array}$ \\
\hline \multicolumn{5}{|c|}{ Horizontal fit } \\
\hline No & 1 & 0 & 4 & - \\
\hline Yes & 9 & 10 & 6 & ID-IDF \\
\hline \multicolumn{5}{|c|}{ Vertical fit } \\
\hline No & 3 & 0 & 3 & - \\
\hline Yes & 7 & 10 & 7 & - \\
\hline \multicolumn{5}{|c|}{ Occlusal fit } \\
\hline Good & 4 & 5 & 3 & - \\
\hline Fair & 3 & 2 & 4 & - \\
\hline Bad & 3 & 3 & 3 & - \\
\hline
\end{tabular}

The interocclusal fit of the crowns did not reveal any statistically significant differences between the impression materials, whereby the results of ID were slightly better than those of IM, and the results of the latter were

18 superior to those of IDF. The duration of the clinical adjustment time of the crowns was found in reverse proportion with the clinical fit. As a result, the time differences required for the dental prosthesis fitting and adjustment in the dentist's chair until the dental prosthesis attained a usable quality could be measured accordingly: on crowns that had been made with the IDF method, the time difference in comparison with ID averaged 38 minutes in comparison with 28 minutes, that is, the difference reached almost significant levels $(p=.0652)$.

\section{DISCUSSION}

\section{Critical Evaluation of the Method}

Contrary to in vitro laboratory studies, clinical studies of impression materials do have the inherent disadvantage that it is difficult to achieve a level of standardization in the impression situs and the impression process. To meet the required levels of standardization to the maximum extent possible, 30 anatomically comparable patient scenarios featuring multiple implants in the side-teeth area were selected.

Moreover, the study was performed in compliance with a standardized dental- and dental technology protocol over a 3 -week period. The differences in the clinical procedures and in the subjective assessments should therefore have been reduced to a minimum. The clinical testing of the precision of the dental prostheses' fit was kept methodically simple and is largely congruent with the regular clinical procedure.

More precise values in reference to distortions in the implant post-impressions can be established through the taking of measurements using measuring bridges with adhesive expanding measuring strips that are periodically utilized in in vitro testing situations.

However, in clinical in vivo test scenarios, the latter do not currently provide solutions for precise applications. Additional laboratory studies are required to precisely investigate the material scientific differences between the chosen impression variants of this study.

\section{Discussion of the Results}

In depicting a combination impression of implants and natural teeth in conjunction with the open impressions technique and a custom tray, all measured clinical dental prosthesis precision parameters demonstrated noninferiority of the new impression material (ID) in comparison with polyether materials (IM).

The combination of a vinylsiloxanether with a polyvinylsiloxane in the IDF group did not yield any additional benefits but actually introduced some disadvantages: although splinting of the implant impression copings as used in the IDF group did give the dentist a sense that the impression quality was superior ( $p=.0151 \mathrm{IDF}$ vs ID or $p=.0024$ IDF vs IM), this was not corroborated by the evaluation of the rotation resistance of the impression copings when the laboratory implants were fixated by the dental technician. The 


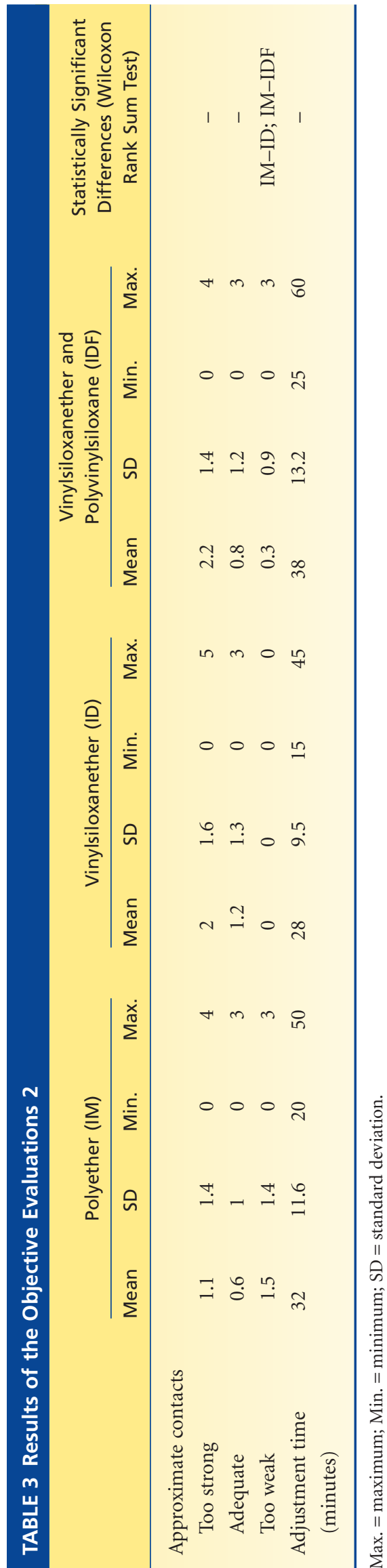

clinical measurements taken of the horizontal dimension displacements between the implant abutments yielded significantly worse results for IDF in comparison with ID ( $p=.0253)$. The slightly worse clinical fits found in the IDF group were also evident in the evaluation of the initial occlusal fits and the time required to make the dental prostheses' fit. Consequently, an excellent clinical perception during the impression's evaluation can be deceiving. In reference to the approximate contacts, the results generated in the ID group and in the IDF group were similarly satisfactory or better than in the IM group. Approximate contact points of the crowns consistently existed in the ID group, that is, all required adjustments could be made right in the dentist's chair. A statistically significant appearance of missing approximate contacts could be determined in the IM group in comparison with the ID group $(p=.0057)$ and in comparison with the IDF group ( $p=.0399)$. This is congruent with the results depicted in the reference literature. The problem of "Impregum" impressions depicting implant clearances that are slightly too small has also been identified in previous studies. ${ }^{13}$ However, in summary, the clinical results obtained in the three impression procedures were all comparably good. Not a single impression-taking process had to be repeated. No differences could be found in terms of the time required for taking the impressions - the determining factor for the time required was the hardening time of the elastomeric masses. The "Futar D Slow" material, which was used as a supplement in the IDF group and added into the tray, did not cause a significant prolongation of the impression session.

One notable finding in reference to the subjective assessments was, in particular, the better taste rating the polyvinyl ether received in comparison with the polyether material. In the clinical process, ID and IDF proved superior to IM in terms of the dentists' assessment of parameters handling and detail precision of the impression, and in terms of the dental technicians' rating in regard to the ease of removal of the impression from the cast mold. In reference to parameter hydrophilic properties and detail precision of the master model, which makes "Impregum" such a popular material with dentists, all three study groups delivered excellent and comparable results.

When comparing parameters detail precision of the impression as rated by the dentist and detail precision of the master model as rated by the dental technician, it is 
striking that the superiority of ID and IDF in the detail precision of the impression is not reflected in an increased detail precision of the model. Instead, the pertinent results were equivalent and excellent overall. The differences in the evaluation of the impression's detail precision can likely be attributed to the fact that because of the lighter coloring of the "Identium," it proved easier to assess than the darker "Impregum." From a dental, clinical point of view, good legibility is an advantage, given that it allows for an expeditious assessment of the impression quality and for the identification of the need to make a new impression.

The significantly better assessment of the handling attained by ID and IDF must be attributed to the material properties that cause them to drip less than IM. These material properties could prove advantageous when utilized in an impression-making process with a metal stock tray that has a larger reservoir for impression material. In the case of "Impregum," it has been proven in the past that the making of an impression with a stiff, custom tray that warrants a layer thickness of 2 to $4 \mathrm{~mm}$ of impression material delivers superior results than one made with a ready-to-use perforated 20 spoon. ${ }^{8,18,30,31}$ A problem of this dimension, which would render the precision level contingent upon the material layer thickness, does not exist with A-silicones: as a result, impressions made with a perforated metal stock tray are as precise as impressions made with a custom tray. ${ }^{34}$ In reference to "Identium" - the new material which from a material science standpoint must be categorized between A-silicones and polyether materials, this issue will have to be newly clarified through experiments and is subject to further studies to be performed in the future.

\section{Clinical Relevance}

Vinylsiloxanether, the new material for impression taking, appears to be a good alternate for polyether materials: based on the results of our study, it allows users to achieve excellent fits for dental prostheses and simultaneously achieves very positive ratings in terms of its clinical handling.

\section{ACKNOWLEDGMENT}

The authors would like to acknowledge the support by

21 Dipl. Stat. Anja Grieger (Langenfeld, Germany) for the statistical evaluation of the study data.

\section{REFERENCES}

1. Spector MR, Donovan TE, Nicholls JI. An evaluation of impression techniques for osseointegrated implants. J Prosthet Dent 1990; 63:444-447.

2. Wee AG, Aquilino SA, Schneider RL. Strategies to achieve fit in implant prosthodontics: a review of the literature. Int J Prosthodont 1999; 12:167-178.

3. Carr AB. Comparison of impression techniques for a fiveimplant mandibular model. Int J Oral Maxillofac Implants 1991; 6:448-455.

4. Heckmann SM, Karl M, Wichmann MG, Winter W, Graef F, Taylor TD. Loading of bone surrounding implants through three-unit fixed partial denture fixation: a finite-element analysis based on in vitro and in vivo strain measurements. Clin Oral Implants Res 2006; 17:345-350.

5. Sahin S, Cehreli MC. The significance of passive framework fit in implant prosthodontics: current status. Implant Dent 2001; 10:85-92.

6. Inturregui JA, Aquilino SA, Ryther JS, Lund PS. Evaluation of three impression techniques for osseointegrated oral implants. J Prosthet Dent 1993; 69:503-509.

7. Humphries RM, Yaman P, Bloem TJ. The accuracy of implant master casts constructed from transfer impressions. Int J Oral Maxillofac Implants 1990; 5:331-336.

8. Boulton JL, Gage JP, Vincent PF, Basford KE. A laboratory study of dimensional changes for three elastomeric impression materials using custom and stock trays. Aust Dent J 1996; 41:398-404.

9. Thongthammachat S, Moore BK, Barco MT, Hovijitra S, Brown DT, Andres CJ. Dimensional accuracy of dental casts: influence of tray material, impression material, and time. J Prosthodont 2002; 11:98-108.

10. Wee AG. Comparison of impression materials for direct multi-implant impressions. J Prosthet Dent 2000; 83:323331.

11. Lorenzoni M, Pertl C, Penkner K, Polansky R, Sedaj B, Wegscheider WA. Comparison of the transfer precision of three different impression materials in combination with transfer caps for the Frialit-2 system. J Oral Rehabil 2000; 27:629-638.

12. Akca K, Cehreli MC. Accuracy of 2 impression techniques for ITI implants. Int J Oral Maxillofac Implants 2004; 19:517-523.

13. Ortorp A, Jemt T, Back T. Photogrammetry and conventional impressions for recording implant positions: a comparative laboratory study. Clin Implant Dent Relat Res 2005; 7:43-50.

14. Conrad HJ, Pesun IJ, DeLong R, Hodges JS. Accuracy of two impression techniques with angulated implants. J Prosthet Dent 2007; 97:349-356.

15. Wenz HJ, Hertrampf K. Accuracy of impressions and casts using different implant impression techniques in a multiimplant system with an internal hex connection. Int J Oral Maxillofac Implants 2008; 23:39-47. 
16. Choi JH, Lim YJ, Yim SH, Kim CW. Evaluation of the accuracy of implant-level impression techniques for internalconnection implant prostheses in parallel and divergent models. Int J Oral Maxillofac Implants 2007; 22:761-768.

17. Daoudi MF, Setchell DJ, Searson LJ. A laboratory investigation of the accuracy of two impression techniques for singletooth implants. Int J Prosthodont 2001; 14:152-158.

18. Burns J, Palmer R, Howe L, Wilson R. Accuracy of open tray implant impressions: an in vitro comparison of stock versus custom trays. J Prosthet Dent 2003; 89:250-255.

19. Naconecy MM, Teixeira ER, Shinkai RS, Frasca LC, Cervieri A. Evaluation of the accuracy of 3 transfer techniques for implant-supported prostheses with multiple abutments. Int J Oral Maxillofac Implants 2004; 19:192-198.

20. Cehreli MC, Akca K. Impression techniques and misfitinduced strains on implant-supported superstructures: an in vitro study. Int J Periodontics Restorative Dent 2006; 26:379-385.

21. Schmitt JK, Adrian ED, Gardner FM, Gaston ML. A comparison of impression techniques for the CeraOne abutment. J Prosthodont 1994; 3:145-148.

22. Eid N. An implant impression technique using a plaster splinting index combined with a silicone impression. J Prosthet Dent 2004; 92:575-577.

23. Assif D, Marshak B, Schmidt A. Accuracy of implant impression techniques. Int J Oral Maxillofac Implants 1996; 11:216-222.

24. Assif D, Nissan J, Varsano I, Singer A. Accuracy of implant impression splinted techniques: effect of splinting material. Int J Oral Maxillofac Implants 1999; 14:885-888.

25. Vigolo P, Majzoub Z, Cordioli G. Evaluation of the accuracy of three techniques used for multiple implant abutment impressions. J Prosthet Dent 2003; 89:186-192.
26. Dumbrigue HB, Gurun DC, Javid NS. Prefabricated acrylic resin bars for splinting implant transfer copings. J Prosthet Dent 2000; 84:108-110.

27. Burawi G, Houston F, Byrne D, Claffey N. A comparison of the dimensional accuracy of the splinted and unsplinted impression techniques for the Bone-Lock implant system. J Prosthet Dent 1997; 77:68-75.

28. De La Cruz JE, Funkenbusch PD, Ercoli C, Moss ME, Graser GN, Tallents RH. Verification jig for implant-supported prostheses: a comparison of standard impressions with verification jigs made of different materials. J Prosthet Dent 2002; 88:329-336.

29. Ormianer Z, Laufer BZ, Nissan J, Gross M. An investigation of heat transfer to the implant-bone interface related to exothermic heat generation during setting of autopolymerizing acrylic resins applied directly to an implant abutment. Int J Oral Maxillofac Implants 2000; 15:837-842.

30. Millstein P, Maya A, Segura C. Determining the accuracy of stock and custom tray impression/casts. J Oral Rehabil 1998; 25:645-648.

31. Valderhaug J, Floystrand F. Dimensional stability of elastomeric impression materials in custom-made and stock trays. J Prosthet Dent 1984; 52:514-517.

32. Feran K, Spencer A. Accuracy and stability for implant impressions. Dent Clin 2007; $\cdots: 30-36$.

33. Rubel BS. Impression materials: a comparative review of impression materials most commonly used in restorative dentistry. Dent Clin North Am 2007; 51:629-642.

34. Tjan AH, Nemetz H, Nguyen LT, Contino R. Effect of tray space on the accuracy of monophasic polyvinylsiloxane impressions. J Prosthet Dent 1992; 68:19-28. 


\begin{tabular}{|l|l|}
\hline \multicolumn{2}{|c|}{ SNP Best-set Typesetter Ltd. } \\
\hline Journal Code: CID & Proofreader: Mony \\
\hline Article No: 240 & Delivery date: 25 August 2009 \\
\hline Page Extent: 8 & Copyeditor: Jenny \\
\hline
\end{tabular}

\section{AUTHOR QUERY FORM}

Dear Author,

During the preparation of your manuscript for publication, the questions listed below have arisen. Please attend to these matters and return this form with your proof.

Many thanks for your assistance.

\begin{tabular}{|c|c|c|}
\hline $\begin{array}{c}\text { Query } \\
\text { References }\end{array}$ & Query & Remark \\
\hline q1 & $\begin{array}{l}\text { AUTHOR: A running head short title was not supplied; please check if this one is suitable } \\
\text { and, if not, please supply a short title that can be used instead. }\end{array}$ & \\
\hline$q^{2}$ & $\begin{array}{l}\text { AUTHOR: Please provide the job titles of all authors (eg, associate professor). Also, please } \\
\text { confirm that the affiliation addresses are correct. }\end{array}$ & \\
\hline$q^{3}$ & AUTHOR: Please confirm that the title of this article is correct. & \\
\hline$q^{4}$ & AUTHOR: Please provide the qualifications of all authors (eg, $\mathrm{PhD}$ ). & \\
\hline $\mathrm{q}^{5}$ & $\begin{array}{l}\text { AUTHOR: The performance ... (IDF). This sentence has been reworded for clarity. Please } \\
\text { check and confirm it is correct. }\end{array}$ & \\
\hline q6 & $\begin{array}{l}\text { AUTHOR: By “. . . of ID, IDF versus IM . . .”, do you mean “. . . of ID, and IDF versus } \\
\text { IM . ..”? If so, please make the necessary changes where applicable. }\end{array}$ & \\
\hline q7 & $\begin{array}{l}\text { AUTHOR: By “. . . made using ID being . . .”, do you mean “. . . made using ID, it } \\
\text { being . ..”? }\end{array}$ & \\
\hline q8 & $\begin{array}{l}\text { AUTHOR: Please note that all instances of "vinyl polysiloxane" have been changed to } \\
\text { "polyvinylsiloxane". Please confirm that these are correct. }\end{array}$ & \\
\hline q9 & $\begin{array}{l}\text { AUTHOR: According to . . . expeditiously. This sentence has been reworded for clarity. } \\
\text { Please check and confirm it is correct. }\end{array}$ & \\
\hline q10 & $\begin{array}{l}\text { AUTHOR: The clinical situation .... taking. This sentence has been reworded for clarity. } \\
\text { Please check and confirm it is correct. }\end{array}$ & \\
\hline q11 & $\begin{array}{l}\text { AUTHOR: Please note that all instances of "occlude stops" have been changed to "occluding } \\
\text { stops". Please confirm that these are ok. }\end{array}$ & \\
\hline q12 & $\begin{array}{l}\text { AUTHOR: Before the tray . . "Impregum." The meaning of this sentence is not clear; please } \\
\text { rewrite or confirm that the sentence is correct. }\end{array}$ & \\
\hline q13 & $\begin{array}{l}\text { AUTHOR: The groups were . . . of the impression. This sentence has been reworded for } \\
\text { clarity. Please check and confirm it is correct. }\end{array}$ & \\
\hline q14 & $\begin{array}{l}\text { AUTHOR: The respective . . . ratings. This sentence has been reworded for clarity. Please } \\
\text { check and confirm it is correct. }\end{array}$ & \\
\hline q15 & $\begin{array}{l}\text { AUTHOR: Please note that citations for Figures and Tables are not allowed in headings. } \\
\text { Thus, all citations for figures and tables found in headings in this article have been moved to } \\
\text { paragraphs following the headings. Please check and confirm that the changes made are ok. }\end{array}$ & \\
\hline q16 & $\begin{array}{l}\text { AUTHOR: No differences .... dental technicians. This sentence has been reworded for } \\
\text { clarity. Please check and confirm it is correct. }\end{array}$ & \\
\hline q17 & $\begin{array}{l}\text { AUTHOR: The post . . . excellent.” This sentence has been reworded for clarity. Please } \\
\text { check and confirm it is correct. }\end{array}$ & \\
\hline
\end{tabular}




\begin{tabular}{|c|c|}
\hline q18 & $\begin{array}{l}\text { AUTHOR: The interocclusal ... IDF. This sentence has been reworded for clarity. Please } \\
\text { check and confirm it is correct. }\end{array}$ \\
\hline q19 & $\begin{array}{l}\text { AUTHOR: More precise values . . . situations. This sentence has been reworded for clarity. } \\
\text { Please check and confirm it is correct. }\end{array}$ \\
\hline $\mathrm{q} 20$ & $\begin{array}{l}\text { AUTHOR: In the case . . . spoon. This sentence has been reworded for clarity. Please check } \\
\text { and confirm it is correct. }\end{array}$ \\
\hline $\mathrm{q} 21$ & AUTHOR: Please provide the full form of "Dipl. Stat." \\
\hline $\mathrm{q} 22$ & $\begin{array}{l}\text { AUTHOR: Please confirm that the abbreviated journal title for Ref. } 32 \text { is correct. Also, } \\
\text { please provide the vol. no. for this reference. }\end{array}$ \\
\hline $\mathrm{q} 23$ & $\begin{array}{l}\text { AUTHOR: Please confirm that the changes made to Tables } 1 \text { to } 3 \text { are ok. Also, please } \\
\text { confirm that the full forms of the abbreviations in Tables } 1 \text { and } 3 \text { are correct. }\end{array}$ \\
\hline
\end{tabular}

